\title{
On Norm-Dependent Positive Definite Functions
}

\author{
By \\ Yasuo YAMASAKI*
}

Summary: Any norm-dependent positive definite function on an infinite dimensional normed space can be written as a superposition of $\exp \left(-c\|\cdot\|^{2}\right)$. Conversely, for a Hilbert space, any superposition of $\exp \left(-c\|\cdot\|^{2}\right)$ is positive definite. A norm-dependent positive definite function exists only if the norm is of cotype 2 . If $\exp \left(-\|\cdot\|^{a}\right)$ is positive definite for some $a_{0}>0$, such $\alpha$ form an interval $\left(0, \alpha_{0}\right]$ where $\alpha_{0} \leqq 2$. If $\alpha_{0}=2$, then $\|\cdot\|$ is a Hilbertian norm. For $\left(l^{p}\right), 0<p \leqq 2$, we have $\alpha_{0}=p$. (Though $\|x\|=\left(\sum_{n}\left|x_{n}\right|^{p}\right)^{1 / p}$ is not a norm for $0<p<1$, the last statement remains valid).

In [1], Chapter 3 , it was shown that on a Hilbert space, any positive definite function dependent only on the norm can be written in the form:

$$
\chi(\xi)=\int_{[0, \infty)} \exp \left(-c\|\xi\|^{2}\right) d \nu(c)
$$

where $\nu$ is a finite measur on $[0, \infty)$. The proof is based on Bernstein's theorem, which claims:

Proposition 1 (Bernstein's Theorem). Let $f(t)$ be a function on $[0, \infty)$. If and only if $f(t)$ is continuous and completely monotone, it is the Laplace transform of a positive measure on $[0, \infty)$, namely it can be written as

$$
f(t)=\int_{[0, \infty)} \exp (-s t) d \nu(s) .
$$

Here, compleie monotoneness is defined as:

Definition 1. A function on $[0, \infty)$ is said to be completely monotone, if for any $t, \tau>0$ and $n=0,1,2, \cdots$ we have

$$
(-1)^{n} \Delta_{\tau}^{n} f(t) \geqq 0
$$

where

$$
\Delta_{\tau} f(t)=f(t+\tau)-f(t) .
$$

Note that if $f(t)$ is known to be infinitely differentiable, complete mono-

Received November 15, 1989.

* Research Institute for Mathematical Sciences, Kyoto University, Kyoto, 606, Japan. 
toneness is characterized by $(-1)^{n}\left(d^{n} / d t^{n}\right) f(t) \geqq 0$.

The proof of Bernstein's theorem, omitted here, can be found for instance in [2], Chapter 4.

For a Hilbert space, if $\chi(\xi)=\varphi\left(\|\xi\|^{2}\right)$ is positive definite, then $\varphi$ must be completely monotone. The proof is given in [1] and also in [3] with some related discussions. But in favor of Dvoretzky's theorem, this statement is kept valid for any infinite dimensional normed space.

Proposition 2 (Dvoretzky's theorem). Let $X$ be an infinite dimensional normed space. For any $\varepsilon>0$ and positive integer $n$, there exist an n-dimensional subspace $R$ and a Hilbertian norm $\|\cdot\|_{H}$ on $R$ such that

$$
(1-\varepsilon)\|\xi\|_{H} \leqq\|\xi\| \leqq(1+\varepsilon)\|\xi\|_{H} \quad \text { for } \quad \forall \xi \in R .
$$

This theorem appeared in [4], and arose many researcher's interest which led to more detailed discussions, for instance [5].

Proposition 3. Let $X$ be an infinite dimensional normed space.

If $\chi(\xi)=\varphi\left(\|\xi\|^{2}\right)$ is continuous and positive definite, $\varphi$ must be completely monotone.

Proof. For given $t_{0}>0$ and $\eta>0$, there exists an $\varepsilon>0$ such that

$$
\left|\varphi(t)-\varphi\left(t_{0}\right)\right| \leqq \eta \quad \text { for } \quad(1-\varepsilon)^{2} t_{0} \leqq t \leqq(1+\varepsilon)^{2} t_{0} .
$$

For this $\varepsilon$ and any given positive integer $n$, there exist an $n$-dimensional subspace $R$ of $X$ and a Hilbertian norm $\|\cdot\|_{H}$ on $R$ which satisfies (5).

Let $\left\{e_{i}\right\}_{i=1}^{n}$ be a CONS of $R$ in $\|\cdot\|_{H}$. Since $\chi$ is positive definite, we have

$$
\sum_{i=1}^{n} \sum_{j=1}^{n} \chi\left(\sqrt{\frac{t_{0}}{2}}\left(e_{i}-e_{j}\right)\right) \geqq 0 \text {. }
$$

For $i \neq j$, we have $\left\|\sqrt{\frac{t_{0}}{2}}\left(e_{i}-e_{j}\right)\right\|_{H}^{2}=t_{0}$, so that $\chi\left(\sqrt{\frac{t_{0}}{2}}\left(e_{i}-e_{j}\right)\right) \leqq \varphi\left(t_{0}\right)+\eta$. Thus we get

$$
n \chi(0)+n(n-1)\left(\varphi\left(t_{0}\right)+\eta\right) \geqq 0,
$$

hence $\varphi\left(t_{0}\right) \geqq-\frac{\chi(0)}{n-1}-\eta$. Since $n>0$ and $\eta>0$ are arbitrary, we must have $\varphi\left(t_{0}\right) \geqq 0$.

Next, for given $t_{0}>0, \tau>0$ and $\eta>0$, we asssume that (6) holds also for $t_{0}+\tau$ instead of $t_{0}$ and that $R$ is $(n+1)$-dimensional and $\left\{e_{i}\right\}_{i=0}^{n}$ is its CONS in $\|\cdot\|_{H}$. Put $\xi_{i}=\sqrt{\frac{t_{0}}{2}} e_{\imath}, \alpha_{2}=1$ for $1 \leqq i \leqq n$ and $\xi_{i}=\sqrt{\frac{t_{0}}{2}} e_{n-i}+\sqrt{\tau} e_{0}, \alpha_{\imath}=-1$ for $n+1 \leqq i \leqq 2 n$. Then, since $\chi$ is positive definite, we have 
(8)

$$
\begin{aligned}
& 0 \leqq \sum_{i, j=1}^{2 n} \alpha_{i} \alpha_{j} \chi\left(\xi_{i}-\xi_{j}\right) \\
& =2 \sum_{i, j=1}^{n}\left[\chi\left(\sqrt{\frac{t_{0}}{2}}\left(e_{i}-e_{j}\right)\right)-\chi\left(\sqrt{\frac{t_{0}}{2}}\left(e_{i}-e_{j}\right) \pm \sqrt{\tau} e_{0}\right)\right] .
\end{aligned}
$$

Thus we get

$$
0 \leqq n \chi(0)+n(n-1)\left(\varphi\left(t_{0}\right)+\eta\right)-n(\varphi(\tau)-\eta)-n(n-1)\left(\varphi\left(t_{0}+\tau\right)-\eta\right),
$$

hence

$$
\varphi\left(t_{0}\right)-\varphi\left(t_{0}+\tau\right) \geqq-\frac{\chi(0)}{n-1}-\eta+\frac{\varphi(\tau)-\eta}{n-1}-\eta
$$

Since $n>0$ and $\eta>0$ are arbitrary, we must have $\varphi\left(t_{0}\right)-\varphi\left(t_{0}+\tau\right) \geqq 0$.

In a similar way, we can prove $(-1)^{m} \Delta_{\tau}^{m} \varphi\left(t_{0}\right) \geqq 0$ for any $m$, hence $\varphi$ is completely monotone.

q.e.d.

Combining the above Proposition 3 with Proposition 1, we obtain the following result.

Proposition 4. Let $X$ be an infinite dimensional normed space. If a positive definite function $\chi(\xi)$ is continuous and depends only on the norm $\|\xi\|$, it is written in the form of (1).

Remark 1. For a Hilbert space, any function $\chi(\xi)$ in the form of (1) is positive definite, but for a general infinite dimensional normed space, the converse is false. Indeed, we know :

Proposition 5. If $\chi(\xi)=\exp \left(-\|\xi\|^{2}\right)$ is positive definite on a normed space $X$, then $X$ must be a Hilbert space.

Proof. By (infinite dimensional) Bochner's theorem (for instance, c.f. [6]), $\chi$ corresponds to a $\sigma$-additive measure $\mu$ on $X^{a}$, the algebraic dual space of $X$. The correspondence is

$$
\chi(\xi)=\int \exp (i x(\xi)) d \mu(x), \xi \in X, x \in X^{a} .
$$

For a fixed $\xi \neq 0$, the equality $\chi(t \xi)=\exp \left(-t^{2}\|\xi\|^{2}\right)$ means that $x(\xi)$ follows onedimensional Gaussian distribution of the variance $2\|\xi\|^{2}$. So that we have

$$
\|\xi\|^{2}=\frac{1}{2} \int x(\xi)^{2} d \mu(x) .
$$

Thus, the function $\Phi_{\xi}(x)=x(\xi)$ belongs to $L^{2}(\mu)$, and the map $\xi \rightarrow \frac{1}{\sqrt{2}} \Phi_{\xi}$ becomes a norm-preserving imbedding of $X$ into $L^{2}(\mu)$. Hence $X$ is a Hilbert space as a subspace of $L^{2}(\mu)$. 
Remark 2. A norm-dependent positive definite function does not always exist. Especially, if the norm is not of cotype 2, it never exists. (cf. [6] Part B Theorem 19.7 and its corollary).

Next, we shall discuss about whether $\exp \left(-\|\cdot\|^{\alpha}\right)$ is positive definite or not. The following results are essentially known ([7], [8]), but we shall formulate and prove them in our way. For a preparation, we state a lemma.

Lemma. On $[0, \infty)$, the function $f_{\alpha}(t)=\exp \left(-t^{\alpha}\right)$ is completely monotone if and only if $0 \leqq \alpha \leqq 1$.

Proof. Evidently $f_{\alpha}(t)$ is not completely monotone for $\alpha<0$. We shall check the sign of $\frac{d^{n} f_{\alpha}}{d t^{n}}$.

$$
\frac{d}{d t} f_{\alpha}=-\alpha t^{\alpha-1} \exp \left(-t^{\alpha}\right) \leqq 0
$$

is all right if $\alpha \geqq 0$.

$$
\frac{d^{2}}{d t^{2}} f_{\alpha}=\left[-\alpha(\alpha-1) t^{\alpha-2}+\alpha^{2} t^{2 \alpha-2}\right] \exp \left(-t^{a}\right) \geqq 0
$$

is true if $0 \leqq \alpha \leqq 1$, but false for sufficiently small $t$ if $\alpha>1$. Suppose that

$$
\frac{d^{n}}{d t^{n}} f_{\alpha}=\sum_{k=1}^{n} a_{k n} t^{k \alpha-n} \exp \left(-t^{\alpha}\right) \text { and }(-1)^{n} a_{k n} \geqq 0 \text { for } 0 \leqq \alpha \leqq 1 \text {. }
$$

Then we have

$$
\frac{d^{n+1}}{d t^{n+1}} f_{\alpha}=\sum_{k=1}^{n}\left[a_{k n}(k \alpha-n) t^{k \alpha-n-1}-a_{k n} \alpha t^{(k+1) a-n-1}\right] \exp \left(-t^{a}\right) .
$$

This means that

$$
\left\{\begin{array}{l}
a_{1, n+1}=a_{1 n}(\alpha-n) \\
a_{k, n+1}=a_{k n}(k \alpha-n)-a_{k-1, n} \alpha(2 \leqq k \leqq n) \\
a_{n+1, n+1}=-a_{n n} \alpha .
\end{array}\right.
$$

Thus, considering $k \leqq n$ and $0 \leqq \alpha \leqq 1$, we get $(-1)^{n+1} a_{k, n+1} \geqq 0$. This assures that $f_{\alpha}(t)$ is completely monotone if $0 \leqq \alpha \leqq 1$.

q.e.d.

Proposition 6. If $\exp \left(-\|\xi\|^{\alpha_{0}}\right)$ is positive definite on a normed space $X$, so is $\exp \left(-\|\xi\|^{\alpha}\right)$ for $0 \leqq \alpha \leqq \alpha_{0}$.

Proof. Since $\exp \left(-t^{\alpha / \alpha_{0}}\right)$ is completely monotone, from Bernstein's theorem we have

$$
\exp \left(-\|\xi\|^{\alpha}\right)=\int_{[0, \infty)} \exp \left(-s\|\xi\|^{\alpha_{0}}\right) d \nu(s)
$$


Since positive definiteness is closed under pointwise convergence and linear combination with positive coefficients, (11) assures that $\exp \left(-\|\xi\|^{\alpha}\right)$ is positive definite.

Remark 3. The set $\left\{\alpha>0 ; \exp \left(-\|\xi\|^{a}\right)\right.$ is positive definite $\}$ forms an interval, if not empty. This interval is closed at right, since positive definiteness is closed under pointwise convergence, so that it is of the form of $\left(0, \alpha_{0}\right]$.

We have $\alpha_{0} \leqq 2$, since every norm-dependent positive definite function is written in the form of (1), and $\exp \left(-t^{a / 2}\right)$ is not completely monotone for $\alpha>2$. We have $\alpha_{0}=2$ if and only if $X$ is a Hilbert space.

Proposition 7. Let $\varphi(\xi)$ be a non-negative function on $X$. Suppose that for any $n, m$ and $t>0, \tau>0$, there exist $\xi_{i}, \xi_{j}^{\prime}(i=1,2, \cdots, n, j=1,2, \cdots, m)$ such that

and

$$
\varphi\left(\xi_{2}-\xi_{j}\right)=t
$$

$$
\begin{aligned}
& \varphi\left( \pm \xi_{j_{1}}^{\prime} \pm \cdots \pm \xi_{j_{k}}^{\prime}+\xi_{\imath}-\xi_{\jmath}\right)=t+k \tau \\
& \quad \text { for } 1 \leqq i \neq j \leqq n, 1 \leqq j_{1}<j_{2}<\cdots<j_{k} \leqq m .
\end{aligned}
$$

Then, every $\varphi(\cdot)$-dependent positive definite function $\chi(\xi)=F(\varphi(\xi))$ is written in the form of

$$
\chi(\xi)=\int_{[0, \infty)} \exp (-s \varphi(\xi)) d \nu(s)
$$

Proof is obtained similarly as the proof of Proposition 3. In this case $\chi(\xi)=F(\varphi(\xi))$ implies that $F$ is completely monotone.

Corollary. For the space $\left(l^{p}\right), 0<p \leqq 2$, every norm-dependent positive de finite function $\chi(\xi)$ is written in the form of

$$
\chi(\xi)=\int_{[0, \infty)} \exp \left(-s\|\xi\|^{p}\right) d \nu(s) .
$$

Remark 4. Conversely, every $\chi(\xi)$ in the form of (13) is positive definite on $\left(l^{p}\right)$, because $\exp \left(-|t|^{p}\right)$ is positive definite on $\boldsymbol{R}$ and $\exp \left(-\|\xi\|^{p}\right)=$ $\Pi_{k=1}^{\infty} \exp \left(-\left|\xi_{k}\right|^{p}\right)$.

Remark 5. The criterion of this corollary shows us that $\exp \left(-\|\xi\|^{p^{\prime}}\right)$ is positive definite if and only if $0<p^{\prime} \leqq p$. Thus we have $\alpha_{0}=p$ for $\left(l^{p}\right), 0<p \leqq 2$. ( $\alpha_{0}$ is of the same meaning as in Remark 3).

The discussions in the proof of Proposition 7 do not require any norm. So, Corollary and Remarks 4 and 5 are valid also for $0<p<1 .\left(\|\xi\|=\left(\sum_{k=1}^{\infty}\left|\xi_{k}\right|^{p}\right)^{1 / p}\right.$, whether it is a norm or not). 


\section{References}

[1] Umemura, Y., Measures on infinite dimensional vector spaces, Publ. RIMS, Kyoto Univ., 1 (1965), 1-47.

[2] Widder, D.V., The Laplace Transform, Chapter 4, Princeton Univ. Press 1946.

[3] Yamasaki, Y., Invariant measure of the infinite dimensional rotation group, Publ. RIMS Kyoto Univ., 8 (1972) 131-140.

[4] Dvoretzky, A., Some results on convex bodies and Banach spaces, Proc. Int. Symp. on linear spaces, Jerusalem 1961, 123-160.

[5] Figiel, T., Lindenstrauss, J. and Milman, V.D., The dimension of almost spherical sections of convex bodies, Acta Mathematica, 139 (1977), 53-94.

[6] Yamasaki, Y., Measures on infinite dimensional spaces World Scientific 1985.

[7] Berg, C. and Forst, G., Potential theory on locally compact Abelian groups, Springer, 1975.

[8] Bretagnolle, J., Dacunha-Castelle, D. and Krivine, J.L., Lois stables et espaces $L^{p}$, Ann. Inst. H. Poincaré (B) 2, (1966), 231-259. 